\title{
Juan de Nova Island
}

National Cancer Institute

\section{Source}

National Cancer Institute. Juan de Nova Island. NCI Thesaurus. Code C123763.

An island in the Mozambique Channel, between Madagascar and Mozambique. 